\title{
Propuesta de un sistema de recolección de agua lluvia en el edificio BACAD 1 de la Escuela Militar de Cadetes “General José María Córdova”
}

\author{
Rodrigo Andrés Bernal Lugo \\ Luis Alejandro Cantor Rodríguez \\ Jhonnatan Castiblanco Costilla \\ Sergio Andrés Blanco Londoño \\ Escuela Militar de Cadetes "General José María Córdova”
}

\begin{abstract}
RESUMEN
En diferentes instituciones existe una gran demanda de agua en actividades de limpieza y mantenimiento, tales como lavado de pisos y fachadas, y riego de plantas. Sin embargo, estas actividades no requieren del uso de agua potable, por lo que su demanda se puede abastecer con agua lluvia. Por lo tanto, este artículo presenta una propuesta para implementar un sistema de recolección de agua lluvia del edificio BACAD 1 de la ESMIC con el objetivo de reducir el consumo de agua potable y, a su vez, contribuir al cuidado del medio ambiente.
\end{abstract}

PALABRAS CLAVE

abastecimiento de agua; agua lluvia; almacenamiento de agua; precipitación; recolección de agua lluvia; reúso de agua.

\section{CITACIÓN}

Bernal Lugo, R., Cantor Rodriguez, L., Castiblanco Costilla, J. \& Blanco Londoño, S. (2019). Propuesta de un sistema de recolección de agua lluvia en el edificio BACAD 1 de la Escuela Militar de Cadetes “General José María Córdova”. Brújula, Semilleros de Investigación, 7 (13). 30-35. https://doi.org/10.21830/23460628.21 


\section{Introducción}

La recolección de agua lluvia fue muy utilizada hasta el momento histórico en que se crearon estructuras para abastecer agua desde la distancia, por ejemplo, el acueducto romano o sistemas modernos de acueducto (Suárez, García y Mosquera, 2006). En la actualidad, algunos de estos sistemas son obsoletos, ya que las fuentes naturales de abastecimiento no cuentan con la misma cantidad y calidad del agua que en el pasado, y es por esto que nuevamente se han empezado a considerar los sistemas de recolección de agua lluvia como una opción para suplir la demanda (León-Agatón, Córdoba-Ruiz y Carreño-Sayago, 2016).

Generalmente, el agua lluvia se recolecta cerca de la fuente, por lo que algunos autores consideran que el agua lluvia es gratis y que los únicos costos son los de inversión inicial asociados con la construcción de las obras de almacenamiento y distribución. De esta manera, el bajo costo del agua lluvia es su principal ventaja frente a los métodos tradicionales de abastecimiento (Unidad de Apoyo Técnico para el Saneamiento Básico del Área Rural [Unatsabar], 2003). Asimismo, la recolección de agua lluvia permite obtener una independencia parcial o incluso total de las redes de suministro público, lo cual reduce el consumo de agua potable y disminuye el pago de los servicios de acueducto y alcantarillado. También disminuye el caudal del alcantarillado pluvial, con lo cual se evita el ingreso de altos volúmenes de agua a los sistemas de drenaje urbano (Estupiñán-Perdomo y Zapata-García, 2010).

Entre las desventajas, se puede destacar, por una parte, que la recolección de agua lluvia depende de la precipitación del lugar, la cual puede ser incierta, especialmente por las transformaciones de los ecosistemas naturales debido en parte al cambio climático, y por otra, los altos costos de inversión inicial del sistema, sobre todo los del tanque de almacenamiento (Palacio-Castañeda, 2010). Sin embargo, la posición geográfica de Colombia es estratégica para implementar sistemas de recolección de agua lluvia, ya que por encontrarse en la línea ecuatorial es privilegiada en producción de agua (entre 500 y 5.000 milímetros anuales dependiendo de la región), lo cual indica que se podría recoger hasta 5.000 litros de agua por metro cuadrado cada año (Estupiñán-Perdomo y Zapata-García, 2010).

En Colombia son pocos los proyectos de gran escala a nivel urbano que han implementado sistemas de recolección de agua lluvia, sin embargo algunos de los que sí han incorporado estos sistemas han recibido certificaciones internacionales por el uso eficiente del agua en su interior, y también han reducido el pago de servicios de acueducto y alcantarillado (ReyesHincapié y Rubio-Cano, 2014). Adicionalmente, la Ley 373 de 1997, de uso eficiente del recurso hídrico, obliga a los proyectos a recolectar agua lluvia, pero su desconocimiento hace que poco se aplique.

Actualmente el Ejército Nacional de Colombia (EJC) tiene una problemática relacionada con los costos de operación de cada una de las unidades que existen alrededor del territorio nacional, especialmente por el pago de servicios de acueducto y alcantarillado. Por esta razón, implementar sistemas de recolección de agua lluvia podría mitigar los gastos relacionados con estos servicios por el uso de agua lluvia en actividades como lavado de zonas comunes y riego de jardines, en las cuales no es de vital importancia utilizar agua potable (Adler, Carmona y Bojalil, 2008). Adicionalmente, la Escuela Militar de Cadetes General José María Córdova (ESMIC) está ubicada en un área en 
la cual las lluvias provocan encharcamientos en las zonas verdes, principalmente en el campo de paradas. Por lo tanto, un sistema de recolección de aguas lluvias también ayudaría a minimizar los encharcamientos, ya que sería almacenada una parte de esta.

El presente artículo tiene como objetivo proponer la implementación de un sistema de recolección de agua lluvia del edificio BACAD 1 de la ESMIC, para proporcionar una posible solución que ayude a reducir el consumo de agua potable, y a su vez, generar un impacto positivo en el medio ambiente.

\section{Materiales y métodos}

Para esta investigación se recolectó información de diferentes fuentes, como planos, documentos internos, observación de campo, consulta de bibliografía y archivos fotográficos (Alzate, 2016). En esta etapa se revisaron los planos arquitectónicos de la ESMIC para identificar las características de sus cubiertas y así seleccionar el edificio más adecuado para implementar un sistema de recolección de agua lluvia. Asimismo, una vez seleccionado el edificio a través de un reconocimiento de campo y la revisión de planos se identificó y caracterizó su red de aguas lluvias. Posteriormente, se buscaron las curvas de intensidad, duración y frecuencia (IDF) de la estación meteorológica más cercana a las instalaciones de la ESMIC, la cual está ubicada dentro de la Universidad Nacional de Colombia. Con esta información se elaboró el diseño del sistema de recolección de agua lluvia, el cual incluyó la creación de los planos de las redes hidráulicas y los tanques de almacenamiento.

A través del cálculo de la cantidad de agua lluvia esperada y de la capacidad de la cubierta para recogerla, se determinó el caudal esperado, los diámetros de las tuberías y el volumen de almacenamiento de los tanques. Para realizar el cálculo del caudal esperado se utilizó la ecuación del método racional $Q=C I A$, donde $Q$ es el caudal esperado, $C$ es el coeficiente de permeabilidad, $I$ es la intensidad de lluvia y $A$ es el área de la cubierta, empleando un $C=1$. Para determinar el diámetro de las tuberías se empleó la ecuación de Manning $V=(1 / n) R^{2 / 3} S^{1 / 2}$, donde $V$ es la velocidad, $R$ es el radio hidráulico, $S$ es la pendiente de la tubería y $n$ es el coeficiente de rugosidad, empleando un $n=0.009$ y una capacidad hidráulica máxima de la tubería del 70 \%.

\section{Resultados y discusión}

La revisión de los planos arquitectónicos de la ESMIC permitió establecer que el edificio BACAD 1 cumplía con las condiciones necesarias para adecuar un sistema de recolección de agua lluvia, principalmente porque facilita la ubicación de los tanques de almacenamiento del sistema sin necesidad de realizar mayores obras. De la observación de campo se determinó que las cubiertas, canales y bajantes existentes en este edificio eran adecuadas y estaban en óptimas condiciones para utilizarlas dentro del sistema de recolección de aguas lluvias, tal como se ilustra en las figura 1 y 2.

Para el diseño del sistema se seleccionó de las curvas IDF un evento de lluvia con duración de 15 minutos, periodo de retorno de dos años, e intensidad de $0,0184 \mathrm{~L} / \mathrm{s} / \mathrm{m}^{2}$ El sistema de recolección fue dividido en dos tanques de acuerdo con la arquitectura del edificio BACAD 1: el tanque 1 se diseñó para un área de cubierta de $726 \mathrm{~m}^{2}$, conectado con los recolectores A, B, y C, mientras que el tanque 2 se diseñó para un área de cubierta de $910 \mathrm{~m}^{2}$, conectado con los recolectores $\mathrm{D}$, E, y F, como se muestra en la figura 3. Los tanques 1 y 2 se estimaron para recolectar un volumen de agua lluvia de 20 y 15 $\mathrm{m}^{3}$ respectivamente. 


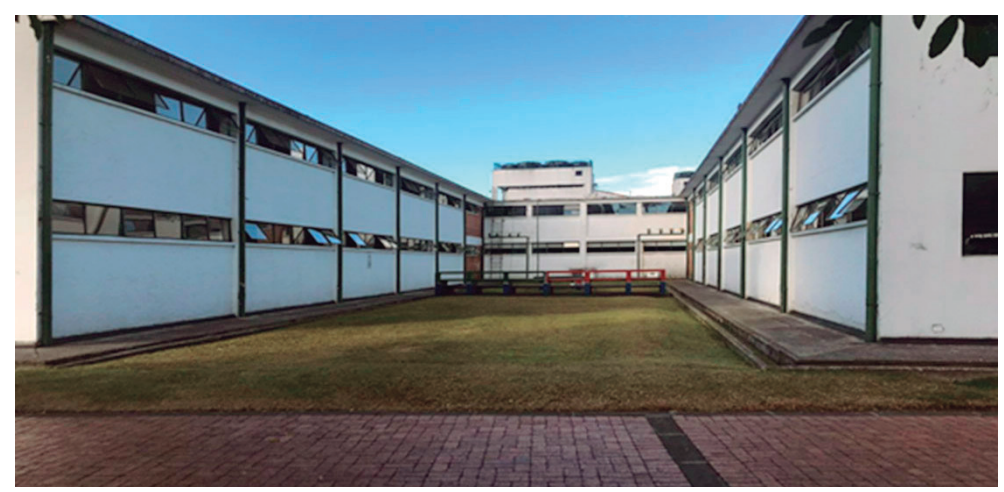

Figura 1. Fachada del edificio BACAD 1.

Fuente: Original de los autores.

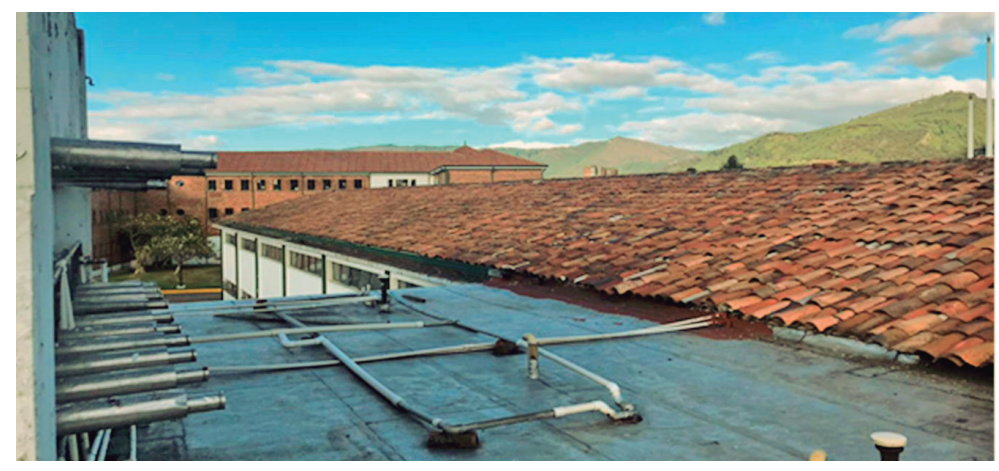

Figura 2. Cubierta del edificio BACAD 1.

Fuente: Original de los autores.

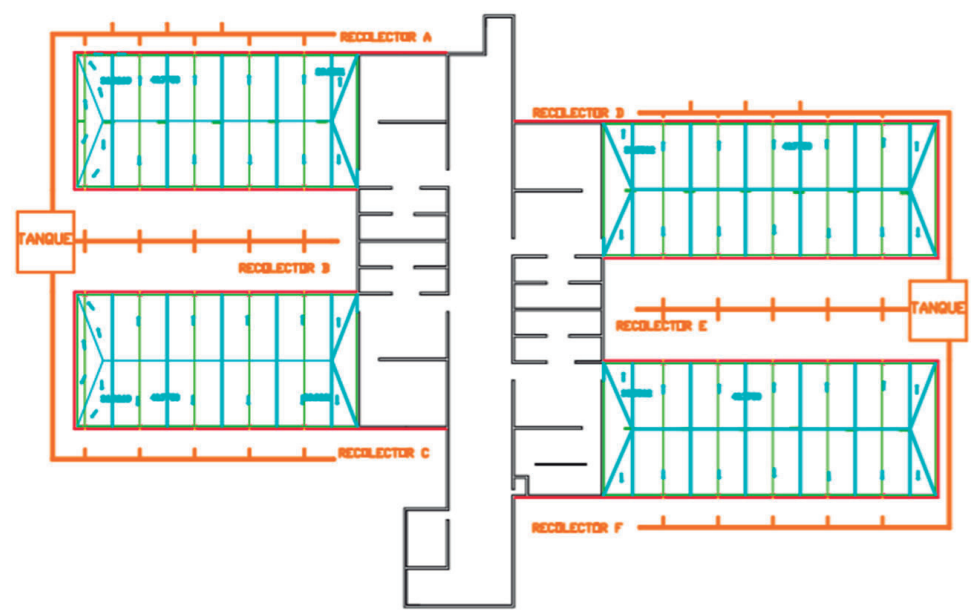

Figura 3. Sistema de recolección de agua lluvia del edificio BACAD 1 de la ESMIC.

Fuente: Original de los autores. 
La demanda de agua para labores de limpieza y mantenimiento por parte de los usuarios del edificio BACAD 1 está relacionada con actividades que se realizan diariamente, como lavado de pisos y riego de plantas, y semanalmente con el lavado de fachadas. Anualmente estas actividades tienen una demanda de $80 \mathrm{~m}^{3}$. Debido a que estas actividades no requieren el uso de agua potable, se estima que el agua lluvia recolectada en el sistema proyectado podría suplir esta demanda. Planteamientos similares han demostrado que la recolección del agua lluvia para labores de limpieza y mantenimiento es una alternativa económica y técnicamente viable y que puede representar una solución para contribuir a una gestión y un desarrollo sostenible de las instituciones (Borrero et al., 2007).

\section{Conclusiones}

Se concluye que es viable implementar un sistema de recolección de agua lluvia para el edificio BACAD 1 de la ESMIC. A mediano y largo plazo, un sistema de recolección de agua lluvia permitirá disminuir los gastos por concepto de pago de servicios de acueducto y alcantarillado que actualmente posee la ESMIC. También permitiría a la Escuela generar un impacto ambiental positivo de acuerdo con las políticas de protección ambiental que tiene el EJC.

\section{Agradecimientos}

Los autores agradecen a la Escuela Militar de Cadetes “General José María Córdova” por el apoyo brindado para la realización de este artículo.

\section{Declaración de divulgación}

Los autores declaran que no existe ningún potencial conflicto de interés relacionado con el texto. Los puntos de vista y los resultados de este artículo pertenecen al autor y no reflejan necesariamente los de la Escuela Militar de Cadetes “General José María Córdova”.

\section{Financiamiento}

Los autores no declaran fuente de financiamiento para la realización de este artículo.

\section{Sobre los autores}

Rodrigo Andrés Bernal Lugo es Alférez de la Escuela Militar de Cadetes, estudiante de la Facultad de Ingeniería Civil, Escuela Militar de Cadetes “General José María Córdova”, Bogotá, D. C., Colombia.

Luis Alejandro Cantor Rodríguez es Alférez de la Escuela Militar de Cadetes, estudiante de la Facultad de Ingeniería Civil, Escuela Militar de Cadetes “General José María Córdova”, Bogotá, D. C., Colombia.

Jhonnatan Castiblanco Costilla es Alférez de la Escuela Militar de Cadetes, estudiante de la Facultad de Ingeniería Civil, Escuela Militar de Cadetes "General José María Córdova”, Bogotá, D. C., Colombia.

Sergio Andrés Blanco Londoño es ingeniero civil y especialista en planeación ambiental y manejo integral de los recursos naturales de la Universidad Militar Nueva Granada y magíster en recursos hidráulicos de la Universidad Nacional de Colombia. Docente en la Facultad de Ingeniería Civil de la Escuela Militar de Cadetes “General José María Córdova”, Bogotá, D. C., Colombia.

\section{Referencias}

Adler, I., Carmona, G. \& Bojalil, J. A. (2008). Manual de captación de aguas de lluvia para centros urbanos. México D. F.: Instituto Internacional del Recursos Renovables de México.

Alzate, L. F. J. O. (2016). Actualización del plano de red y catastro de la Escuela Militar de Cadetes General José María Córdova. Bogotá. 
Borrero, J. A. L., Abello, A. E. T., Pinilla, M. C. C., Castro, L. D., Robayo, J. I. E. \& González, P. A. V. (2007). Aprovechamiento del agua lluvia para riego y lavado de zonas duras y fachadas en el campus de la Pontificia Universidad Javeriana (Bogotá). Ingeniería y Universidad, 11 (2), 193-202.

Estupiñán-Perdomo, J. L. \& Zapata-García, H. O. (2010). Requerimientos de infraestructura para el aprovechamiento sostenible del agua lluvia en el campus de la Pontificia Universidad Javeriana, sede Bogotá (Tesis de Maestría). Universidad Javeriana, Facultad de Ingeniería. Bogotá, D. C., Colombia.

León-Agatón, A., Córdoba-Ruiz, J. C. \& Carreño-Sayago, U. F. (2016). Revisión del estado de arte en captación y aprovechamiento de aguas lluvias en zonas urbanas y aeropuertos. Tecnura, 20 (50), 141-153.

Palacio-Castañeda, N. (2010). Propuesta de un sistema de aprovechamiento de agua lluvia como alternativa para el ahorro de agua potable en la institución educativa María Auxiliadora de Caldas, Antioquia. Revista Gestión y Ambiente, 13(2), 25-40.

Reyes-Hincapié, M. C. \& Rubio-Cano, J. J. (2014). Descripción de los sistemas de recolección y aprovechamiento de aguas lluvias (Tesis de Especialización). Universidad Católica de Colombia, Facultad de Ingeniería. Bogotá D. C., Colombia.

Suárez, J., García, M. \& Mosquera, R. (2006). Historia de los sistemas de aprovechamiento de agua lluvia. VI SEREA. Seminario Iberoamericano sobre Sistemas de Abastecimiento Urbano de Agua. João Pessoa (Brasil), 5.

Unidad de Apoyo Técnico para el Saneamiento Básico del Área Rural [Unatsabar]. (2003). Captación de agua de lluvia para el consumo humano: especificaciones técnicas. Lima: Cepis. Recuperado de https://www.ministeriodesalud.go.cr/index.php/ investigacion-y-tecnologia-en-salud/inventarios/ inventario-tecn-de-agua-de-consumo-humano/ captacion-de-agua-para-consumo-humano/ captando-agua-de-la-lluvia/documento-tecnico-1/1836-captacion-de-agua-de-lluvia-para-consumo-humano/file 